EESTI NSV TEADUSTE AKADEEMIA TOIMETISED. X KOIDE

FOOSIKALIS-MATEMAATILISTE JA TEHNILISTE TEADUSTE SEERIA. 1961, NR. 3

ИЗВЕСТИЯ АКАДЕМИИ НАУК ЭСТОНСКОИ ССР. ТОМ Х СЕРИЯ ФИЗИКО-МАТЕМАТИЧЕСКИХ И ТЕХНИЧЕСКИХ НАУК, 1961, № 3

\title{
О ПРИМЕНИМОСТИ МЕТОДА РАСЧЛЕНЕНИЯ НАПРЯЖЕННОГО СОСТОЯНИЯ ПРИ РЕШЕНИИ ОСЕСИММЕТРИЧЕСКИХ ЗАДАЧ ДИНАМИКИ ЗАМКНУТОЙ ЦИЛИНДРИЧЕСКОЙ ОБОЛОЧКИ
}

\section{н. Алумяэ}

В работе рассматривается неустановившееся колебание полубесконечной круговой замкнутой цилиндрической оболочки под действием краевой нагрузки, изменяющейся во времени по синусоидальному закону. Цель исследования заключается в выявлении основных закономерностей колебания, проявившихся в начальной стадии движения в течение первых периодов изменения нагрузки.

При весьма медленном приложении осесимметрической нагрузки напряженное состояние широкого класса оболочек вращения может быть разложено на безмоментное состояние и краевые эффекты. При быстром же нагружении появляются (нестационарные) волновые процессы, которые в оболочках практически не изучены.*

За математическую модель оболочки принимается система линейных дифференциальных уравнений (1.1) - (1.3) гиперболического типа. Эти уравнения были предложены уже в 1956 году, однако до сих пор при помощи этих уравнений были определены лишь фазовые и групповые скорости распространения упругих возмущений.

Представляет, конечно, интерес установить пределы применимости упрощенных уравнений колебаний для изучения быстропротекающщх процессов в оболочке, нбо расчет по уравнениям гиперболического типа значительно сложнее, чем по теории, базирующейся на гипотезе Кирхгоффа-Лява. Настоящая работа посвящена именно этой теме. Объектом исследования служит цилиндрическая оболочка, в случае которой для решения возможно применение преобразования Лапласа.

Основной вывод исследования заключается в следующем. Если 1) период изменения нагрузки больше или соизмерим со временем, в течение которого упругая волна покрывает расстояние, равное радиусу срединной поверхности оболочки, и 2) узкой целью работы является определение наибольших перемещений и напряжений, то для решения этой задачи возможно расчленение общего напряженного состояния на безмоментное состояние и краевой эффект; эти элементарные состояния могут быть определены упрощенными расчетными соотношениями, известными из теории равновесия, и дополненными инерционными членами.

Это положение нередко допускает простое раздельное решенне смешанных задач (с начальными и краевыми условиями) осесимметрнческого колебания оболочки, по которому (как и в статике) сначала решается безмоментная задача, а затем определяется краевой эффект.

Уравнения гнперболического типа при тонких оболочках практически необходимы лишь в случае, когда период изменения нагрузки очень короток. В предельном (с точки зрения теории оболочек) случае, когда этот период соизмерим со временем про-

* В то же время решены некоторые нелинейные динамические задачи упругих оболочек $\left[{ }^{1-6}\right]$, где задача методом Галеркина сводится к изучению динамики нелинейной снстемы с одной или несколькими степенями свободы. 
хождения упругой волной расстояния, равного толщине стенки оболочки, никакая двумерная модель не может, конечно, правильно описать колебание тонкой оболочки в начальной стадии движения.

1. Основные соотношения. Рассмотрим кругоцилиндрическую замкнутую оболочку с радиусом срединной поверхности $R$ и толщиной $h=$ const. Пусть будет $E-$ модуль упругости; $v-$ коэффициент Пуассона; $\mu$ - плотность материала оболочки; $x$ - расстояние некоторой точки оболочки от торцевого сечения, перпендикулярного оси оболочки; $z R-$ расстояние точки от срединной поверхности оболочки; $t-$ время. Перемещения при осесимметрическом движении зададим в форме

$$
\mathbf{u}=[u(x, t)+z v(x, t)] \mathbf{e}_{x}+w(x, t) \mathbf{e}_{n},
$$

где $\mathbf{e}_{x}, \mathbf{e}_{n}-$ единичные векторы в направлении образующей и нормали $\mathrm{k}$ срединной поверхности.

При отсутствии внешней нагрузки на боковых поверхностях оболочек простейшие уравнения гиперболического типа осесимметрического движения кругоцилиндрической оболочки выражаются в форме

$$
\begin{gathered}
\frac{\partial^{2} u}{\partial a^{2}}-\frac{\partial^{2} u}{\partial \tau^{2}}-v \frac{\partial w}{\partial c}=0 \\
\nu \frac{\partial u}{\partial \alpha}+k^{2} \frac{\partial^{2} w}{\partial \alpha^{2}}-\frac{\partial^{2} w}{\partial \tau^{2}}+\frac{k^{2}}{\varepsilon} \frac{\partial v}{\partial \alpha}=0 \\
-\frac{k^{2}}{\varepsilon} \frac{\partial w}{\partial a}+\frac{\partial^{2} v}{\partial c^{2}}-\frac{\partial 2 v}{\partial \tau^{2}}-\frac{k^{2}}{\varepsilon^{2}} v=0
\end{gathered}
$$

где $\alpha, \tau-$ безразмерные координата и время

$$
\alpha=\frac{x}{R}, \quad \tau=\left(\frac{E}{\left(1-v^{2}\right) \mu}\right)^{1 / 2} \frac{t}{R}, \quad k^{2}=\frac{5(1-v)}{12}, \quad \varepsilon^{2}=\frac{h^{2}}{12 R^{2}} .
$$

Предположим, что на краю оболочки действуют продольная сила $P(\tau)$, изгибаюший момент $M(\tau)$ и поперечная сила $Q(\tau)$. Соответствующие системе (1.1) - (1.3) краевые условия получаются следующие:

$$
\begin{aligned}
\frac{\partial u}{\partial a}-v w & =p(\tau) \\
k^{2}\left(\frac{\partial w}{\partial a}+\frac{1}{\varepsilon} v\right) & =q(\tau) \\
\frac{\partial v}{\partial \alpha} & =m(\tau) .
\end{aligned}
$$

Здесь

$$
p=\left(1-v^{2}\right) \frac{P}{E h}, \quad q=\left(1-v^{2}\right) \frac{Q}{E h}, \quad m=\left(1-v^{2}\right) \frac{M}{E R h} .
$$

Система $(1.1)-(1.3)$ была использована И. Германом и Г. Мирским [7] для определения фазовых скоростей распространения упругих волн по первой форме; добиваясь, чтобы скорость при весьма короткой длине волны была такая же, как получится при трехмерной теории для 
коротких волн, они приняли для параметра $k^{2}$ значение $k^{2}=0,86$ (при $v=0,3)$.

В настоящей работе будет рассмотрена весьма узкая задача: автор ограничивается изучением начальной (по времени) стадии динамики длинной (полубесконечной) оболочки, на краю $\alpha=0$ которой действуют силы

$$
p(\tau)=p_{0} \sin x \tau, \quad q(\tau)=q_{0} \sin \chi \tau, \quad m(\tau) \equiv 0 ;
$$

предполагается, что при $\tau=0$ оболочка находится в покое, т. е.

$$
\begin{gathered}
u(\alpha, 0)=v(\alpha, 0)=w(\alpha, 0)=0 \\
\frac{\partial}{\partial \tau} u(\alpha, 0)=\frac{\partial}{\partial \tau} v(\alpha, 0)=\frac{\partial}{\partial \tau} w(\alpha, 0)=0 .
\end{gathered}
$$

2. Представление решения контурным ингегралом. При помощи преобразования Лапласа получим решение задачи в форме

$w(\alpha, \tau)=\frac{1}{2 i \pi} \int_{L} \sum_{j=1}^{3}\left\{G_{p}(s) F_{j p}(s)+G_{q}(s) F_{j q}(s)\right\} e^{\alpha r_{j}(s)+s \tau} H(s) d s$,

где $G_{p}(s), G_{q}(s)$ - изображения функции $p(\tau), q(\tau)$

$$
G_{p}=p_{0} G(s), \quad G_{q}=q_{0} G(s), \quad G(s)=\frac{\varkappa}{s^{2}+\varkappa^{2}} .
$$

Подинтегральные функции $F_{j p}(s), F_{j q}(s), H(s)$ зависят от корней $r(s)$ уравнения

$$
\begin{gathered}
\varepsilon^{2} k^{2} r^{6}-\left[\left(1+2 k^{2}\right) s^{2}+1-v^{2}\right] \varepsilon^{2} r^{4}+ \\
+\left[\varepsilon^{2}\left(2+k^{2}\right) s^{4}+k^{2}\left(s^{2}+1-v^{2}\right)\right] r^{2}-\varepsilon^{2} s^{6}-k^{2} s^{2}\left(s^{2}+1\right)=0 .
\end{gathered}
$$

Условиям задачи удовлетворяют только три корня $r_{j}(s)$, такие, у которых вещественная часть отрицательна при больших положительных значениях Res; указанные корни описывают распространение возмущений от $\alpha=0$ в положительном направлении $\alpha$. Для индивидуализации дадим их приближенные выражения при больших положительных значениях $s$ :

$$
\begin{aligned}
r_{1}=-s-\frac{v^{2}}{2 s}, \quad r_{2} & =-\frac{1}{k} s+\frac{k^{3}}{2 \varepsilon^{2}\left(1-k^{2}\right) s} \\
r_{3} & =-s-\frac{k^{2}}{2 \varepsilon^{2}\left(1-k^{2}\right) s} .
\end{aligned}
$$

Через корни $r_{1}(s), r_{2}(s), r_{3}(s)$ функции $F_{j p}(s), F_{j q}(s)$. и $H(s)$ выражаются в виде:

$$
\begin{aligned}
& F_{1 p}=-\frac{v r_{2} r_{3}\left(r_{2} r_{3}+s^{2}\right)}{\left(r_{1}-r_{2}\right)\left(r_{3}-r_{1}\right)}\left[1-\frac{\varepsilon^{2}}{k^{2}}\left(r_{1}^{2}-s^{2}\right)\right] \\
& F_{1 q}=-\frac{\left(r_{2}+r_{3}\right)\left(r_{1}^{2}-s^{2}\right)^{2}}{\left(r_{1}-r_{2}\right)\left(r_{3}-r_{1}\right)}\left(1+\frac{\varepsilon^{2}}{k^{2}} s^{2}\right)
\end{aligned}
$$




$$
\begin{aligned}
& F_{2 p}=-\frac{v r_{3} r_{1}\left(r_{3} r_{1}+s^{2}\right)}{\left(r_{2}-r_{3}\right)\left(r_{1}-r_{2}\right)}\left[1-\frac{\varepsilon^{2}}{k^{2}}\left(r_{2}^{2}-s^{2}\right)\right] \quad F_{3 p}=\ldots \\
& F_{2 q}=-\frac{\left(r_{3}+r_{1}\right)\left(r_{2}^{2}-s^{2}\right)^{2}}{\left(r_{2}-r_{3}\right)\left(r_{1}-r_{2}\right)}\left(1+\frac{\varepsilon^{2}}{k^{2}} s^{2}\right) \quad F_{3 q}=\ldots \\
& H(s)=\left\{\left(\frac{\varepsilon^{2}}{k^{2}} s^{4}+s^{2}+1-v^{2}\right)\left(2 s^{2}+r_{1} r_{2}+r_{2} r_{3}+r_{3} r_{1}\right)+\right. \\
& \left.+s^{2}\left[v^{2}+\varepsilon^{2}\left(r_{1}+r_{2}+r_{3}\right) r_{1} r_{2} r_{3}\right]\right\}^{-1} \text {. }
\end{aligned}
$$

Подинтегральное выражение в (2.1) имеет простые полюсы в точках $s= \pm i x$ и значительное число точек разветвления

$$
\begin{gathered}
s= \pm \frac{2 k^{2}}{\left(1-k^{2}\right) \varepsilon}, \quad s= \pm \frac{k}{\varepsilon} i, \quad s= \pm i \\
s= \pm\left\{\sqrt{1-v^{2}} i+3 \cdot 2^{-5 / 3} \varepsilon^{2 / 3} v^{4 / 3}\left(1-\nu^{2}\right)^{1 / 6} e^{i(-\pi / 6+j \cdot 2 \pi / 3)}\right\} \quad(j=0,1,2)
\end{gathered}
$$

На фиг. 1 показаны срезы плоскости $s$, которые необходимы для однозначного определения функций $r_{1}(s), r_{2}(s), r_{3}(s)$. Контур интегрирования $L$ должен быть выбран так, чтобы подинтегральная функция в (2.1) осталась однозначной и аналитической справа от контура. Представленный на фиг. 1 контур $C$ этому условию как будто не удовлетво-

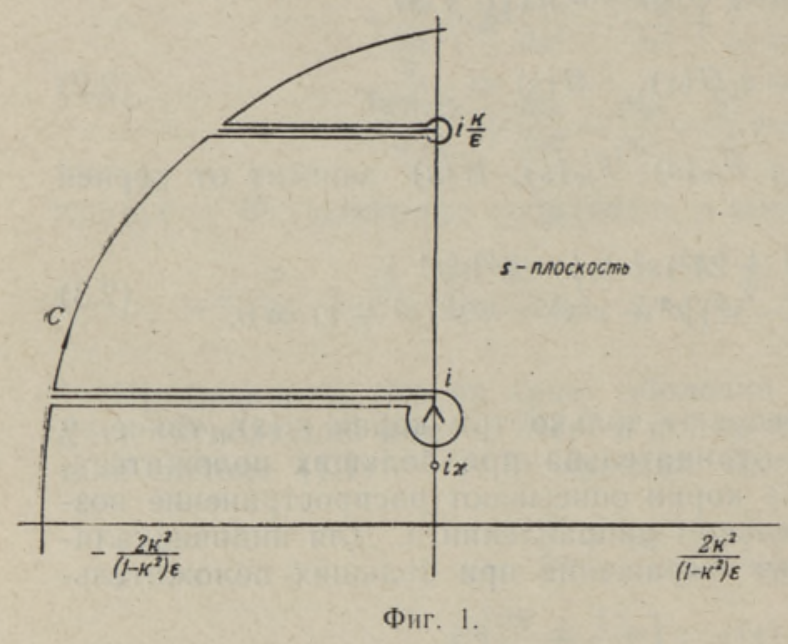

ряет, так как в окаймленной им правой подобласти имеется срез между точками разветвления $s= \pm 2 k^{2} /\left(1-k^{2}\right) \varepsilon$. Одна ко, подинтегральные выражения на верхнем и нижнем берегу среза равны, поэтому значение функции $w(\alpha, \tau)$ не изменяется, если контур интегрирования этот срез не оставит влево от себя *

Остается показать, что подинтегральная функция в (2.1) не имеет полюсов справа от контура $C$ кроме полюсов в точках $s= \pm i$. Из энергетических соотношений задачи следует, что функция $H(s)$ может иметь полюсы лишь при чисто мнимых значениях $s$ : В этом случае доказать требуемое несложно; исключением является только исследование вопроса на малых отрезках мнимой оси около точек $s= \pm i \sqrt{1-v^{2}}$. Но так как контур $C$ все равно оставит эти отрезки слева от себя, можно от доказательства и отказаться. Итак, если точки $s= \pm i x$ находятся справа от контура $C$, то имеет место формула**

* Это утверждение относится не к отдельным слагаемым $\left\{G_{p} F_{j p}+G_{q} F_{j q}\right\} H \times$ $\times \exp \left(\alpha r_{j}+\tau s\right)$ подинтегрального выражения (2.1) при $j=2$, 3, а только к их сумме.

** Если же точки $s= \pm i x$ находятся слева от контура $C$, то вторую группу слагаемых (вне знака интегрирования) учитывать не следует. 


$$
\begin{aligned}
w(\alpha, \tau)= & \frac{1}{2 i \pi} \int_{C} \sum_{j=1}^{3} G(s)\left\{p_{0} F_{j p}(s)+q_{0} F_{j q}(s)\right\} e^{\alpha r_{j}(s)+s \tau} H(s) d s+ \\
& +\frac{1}{2 i} \sum_{j=1}^{3}\left\{\left[p_{0} F_{j p}(i x)+q_{0} F_{j q}(i x)\right] H(i x) e^{\alpha r_{j}(i x)+i x \tau}-\right. \\
& \left.-\left[p_{0} F_{j p}(-i x)+q_{0} F_{j q}(-i x)\right] H(-i x) e^{\alpha r_{j}(-i x)-i x \tau}\right\} .
\end{aligned}
$$

Нетрудно проверить, что вторая группа слагаемых представляет собой стационарное решение задачи - решение в форме $w(\alpha, \tau)=w(\alpha) \sin x \tau$, удовлетворяюшее уравнениям (1.1) - (1.3) и краевым условиям (1.4), (1.5), но не начальным условиям; контурный же интеграл в (2.8) корректирует это решение.

Введем обозначение

$$
I_{i q}(\alpha, \tau)=\int_{L} G(s) F_{i q}(s) H(s) e^{\alpha r_{j}(s)+s \tau} d s .
$$

Нетрудно убедиться, что $I_{1 q}(\alpha, \tau) \equiv 0, \quad I_{3 q}(\alpha, \tau) \equiv 0$ тогда, когда $\alpha>\tau$, а $I_{2 q}(\alpha, \tau) \equiv 0$, когда $\alpha>k \tau$. Отсюда следует, что возмущения распространяются в осевом направлении с двумя скоростями

$$
c_{1}=c_{3}=\sqrt{E /\left(1-v^{2}\right) \mu}, \quad c_{2}=k \sqrt{E /\left(1-v^{2}\right) \mu .}
$$

3. Оценки для функций $r_{j}(s), \quad F_{j}(s), \quad G(s) H(s)$. Пусть будет $s=\sigma e^{i \varphi}, r_{j}(s)=\varrho_{j} e^{i \psi}$. При весьма больших значениях $\sigma\left(\sigma>\varepsilon^{-1}\right)$ будут $\varrho_{j}$ соизмеримы с $\sigma\left(\varrho_{j} \sim \sigma\right)$, причем $r_{1}$ определяется из упрощенного уравнения

$$
r_{1}^{2}=s^{2}+\frac{v^{2} s^{2}}{s^{2}+1-v^{2}}
$$

$r_{2}, r_{3}-$ из уравнения

$$
r^{4}-\frac{1+k^{2}}{k^{2}} s^{2} r^{2}+\frac{1}{k^{2}} s^{4}+\frac{1}{\varepsilon^{2}}\left(s^{2}+1-v^{2}\right)=0 .
$$

При больших значениях $\sigma\left(\varepsilon^{0}<\sigma<\varepsilon^{-1}\right)$ имеем

$$
\mathrm{Q}_{1} \sim \sigma, \quad \mathrm{Q}_{2} \sim \sqrt{\sigma / \varepsilon}, \quad \mathrm{Q}_{3} \sim \sqrt{\sigma / \varepsilon},
$$

за исключением оценки $\varrho_{3}$ в окрестности точек $s=+k i / \varepsilon$ (в этих точках $\left.r_{3}=0\right)$. При малых же значениях $\sigma\left(0<\sigma<\varepsilon^{0}\right)$ найдем

$$
\mathrm{Q}_{1} \sim \sigma, \quad \mathrm{Q}_{2} \sim 1 / \sqrt{\varepsilon}, \quad \mathrm{Q}_{3} \sim 1 / \sqrt{\varepsilon} .
$$

Здесь $r_{1}$ может быть по-прежнему определен формулой (3.1), a $r_{2}, r_{3}$ - упрошенным уравнением (3.2)

$$
r^{4}+\frac{1}{\varepsilon^{2}}\left(s^{2}+1-v^{2}\right)=0
$$

за исключением некоторых окрестностей точек $s= \pm i \sqrt{1-v^{2}}$, где упрощенные формулы неприменимы *.

* В окрестности точек $s= \pm i \sqrt{1-v^{2}}$, где имеются по три точки разветвления корней уравнения (2.2), функции $r_{j}$ изменяются относительно быстро и по модулю, ft по фазе. 
Переходим к оценке величин $G(s) F_{j p}(s) H(s)$ и $G(s) F_{j q}(s) H(s)$, обозначая их ради краткости через $\kappa_{j p}(s)$ и $\kappa_{j q}(s)$ соответственно. Если предположим, что $x \lesssim \varepsilon^{0}$, то на основании формул $(2.5),(2.6)$ и приведенных выше оценок получим:

при весьма больших значениях $\sigma\left(\sigma>\varepsilon^{-1}\right)$

$$
\begin{gathered}
\left|\kappa_{1 p}(s)\right| \sim\left|\kappa_{2 p}(s)\right| \sim\left|\kappa_{3 p}(s)\right| \sim \sigma^{-4} \\
\left|\kappa_{1 q}(s)\right| \sim \varepsilon^{2} \sigma^{-5}, \quad\left|\kappa_{2 q}(s)\right| \sim \sigma^{-3}, \quad\left|\kappa_{3 q}(s)\right| \sim \varepsilon^{-2} \sigma^{-5} ;
\end{gathered}
$$

при больших значениях $\sigma\left(\varepsilon^{0}<\sigma<\varepsilon^{-1}\right)$

$$
\begin{aligned}
& \left|\kappa_{1 p}(s)\right| \sim \sigma^{-4}, \quad\left|\kappa_{2 p}(s)\right| \sim\left|\kappa_{3 p}(s)\right| \sim \varepsilon \sigma^{-3} \\
& \left|\kappa_{1 q}(s)\right| \sim \varepsilon^{2 / 2} \sigma^{-41 / 2}, \quad\left|\kappa_{2 q}(s)\right| \sim\left|\kappa_{3 q}(s)\right| \sim \varepsilon^{-1 / 2} \sigma^{-7 / 2}
\end{aligned}
$$

и, наконец, при малых значениях $\sigma\left(0<\sigma<\varepsilon^{0}\right)$, за исключением неко-

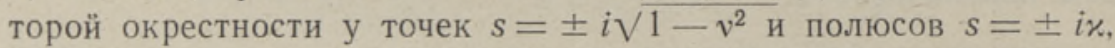

$$
\begin{aligned}
& \left|\kappa_{1 p}(s)\right| \sim \varepsilon^{0}, \quad\left|\kappa_{2 p}(s)\right| \sim\left|\kappa_{3 p}(s)\right| \sim \varepsilon \\
& \left|\kappa_{1 q}(s)\right| \sim \varepsilon^{8 / 2}, \quad\left|\kappa_{2 q}(s)\right| \sim\left|\kappa_{3 q}(s)\right| \sim \varepsilon^{-1 / 2} .
\end{aligned}
$$

Эти оценки могут быть использованы при выводе упрощенных расчетных формул для определения прогиба

4. Прогиб края оболочки. Определение функции $w(\alpha, \tau)$ вдоль линии $\alpha=0$ облегчается тем, что вместо трех экспоненциальных множителей $\exp \left[\alpha r_{j}(s)+\tau s\right]$ в $(2.8)$ имеется только один и при этом весьма простой - $\exp (\tau s)$. Если $\tau$ достаточно большая, то подинтегральная функция изменяется приблизительно как функция $e^{\tau s}$ аргумента $s$, а из этого уже вытекает, что подинтегральная функция в (2.8) принимает наибольшие значения около мнимой оси. Учитывая еще, что по оценкам (3.7), (3.8) $\left|\kappa_{j p}(s)\right|,\left|\kappa_{j q}(s)\right|$ будут при $s \sim i \varepsilon^{0}$ значительно больше, чем при $s \sim i \varepsilon^{-1}$, можно заключить, что контурный интеграл определяется в основном значениями подинтегральной функции по контуру $C$ при $s \sim \varepsilon^{0}$. Вместе с тем, при значениях $s \sim \varepsilon^{0}$ выражения для функций $\kappa_{j p}(s), \kappa_{j q}(s)$ могут быть упрощены. А именно, с погрешностью порядка $\varepsilon$ можно принять

$$
\begin{aligned}
& H(s) \sum_{j=r}^{3} F_{j p}(s)=\frac{v}{s^{2}+1-v^{2}} \\
& H(s) \sum_{j=1}^{3} F_{j q}(s)=\frac{r_{2}+r_{3}}{s^{2}+1-v^{2}},
\end{aligned}
$$

где $r_{2}(s)$ и $r_{3}(s)$ определяются из упрощенного уравнения

$$
\varepsilon^{2} r^{4}+s^{2}+1-v^{2}=0 .
$$

Выбор корней требует, конечно, пояснения. Согласно определению корней $r_{2}$ и $r_{3}$ формулами (2.4), $r_{2}(s)$ на верхнем берегу среза $(-\infty+i, i)$ будет такой корень уравнения (4.3), у которого вещественная часть положительна, а мнимая - отрицательна; на нижнем берегу этого среза и вещественная и мнимая части будут отрицательны. На этом контуре будет $r_{3}(s)=-i r_{2}(s)$. Для определения корней на нижней полуплоскости $s$ (вдоль среза - $-i,-i$ ) отметим, что комплексно сопряженным значениям $s$ соответствуют комплексно сопряженные значения $r_{2}(s)$ и $r_{3}(s)$, т. е. $r_{2}(\bar{s})=\overline{r_{2}(s)}, r_{3}(\bar{s})=\overline{r_{3}(s)}$. 
Упрощенные соотношения могут быть применены для определения функции $w(\alpha, \tau)$ и при некоторой краевой зоне, где $\alpha / \tau$ достаточно мало. Последнее положение будет уточнено ниже, здесь же дадим развернутые расчетные формулы. Пусть будут

$$
\begin{array}{rlrl}
s_{\sigma}(\varphi) & =i \sqrt{1-v^{2}}+\sigma e^{i \varphi}, \quad \sigma=1-\sqrt{1-v^{2},} \quad-\frac{3}{2} \pi \leqslant \varphi \leqslant \frac{1}{2} \pi \\
r_{1}(\varphi) & =-s_{\sigma}\left(\frac{s_{\sigma}^{2}+1}{s_{\sigma}^{2}+1-v^{2}}\right)^{1 / 2}, \quad & \operatorname{Im} r_{1}(\varphi)<0 \\
r_{2}(\varphi) & =\frac{1}{\sqrt{\varepsilon}}\left(-s_{\sigma}^{2}-1+v^{2}\right)^{1 / 4}, & \operatorname{Rer}_{2}(\varphi)<0, \quad \operatorname{Im} r_{2}(\varphi)<0 \\
s_{u} & =s(u)=i-u \\
r_{2}(u) & =\frac{1}{\sqrt{\varepsilon}}\left(-s_{u}^{2}-1+v^{2}\right)^{1 / 4}, & \operatorname{Re}_{2}(u)>0, \quad \operatorname{Im}_{2}(u)<0,
\end{array}
$$

тогда, если $x<i\left(\sqrt{1-v^{2}}-\sigma\right)$, получим

$$
\begin{aligned}
& \omega(\alpha, \tau)=\frac{1}{\pi} p_{0} \nu \varkappa \sigma \operatorname{Re}\left\{\int_{-3 \pi / 2}^{\pi / 2} \frac{\exp \left[a r_{1}(\varphi)+\tau s_{\sigma}+i \varphi\right]}{\left(s_{\sigma}^{2}+x^{2}\right)\left(s_{\sigma}^{2}+1-v^{2}\right)} d \varphi\right\} \\
& +\frac{v p_{\sigma}}{1-v^{2}-x^{2}} \sin x\left[\tau-a\left(\frac{1-x^{2}}{1-v^{2}-x^{2}}\right)^{1 / 2}\right]+ \\
& +\frac{1}{\sqrt{2} \pi} q_{0} \varkappa \sigma \operatorname{Im}\left\{e ^ { i \frac { \pi } { 4 } } \int _ { - 3 \pi / 2 } ^ { \pi / 2 } \frac { r _ { 2 } ( \varphi ) \operatorname { e x p } [ \tau s _ { \sigma } + i \varphi ] } { ( s _ { \sigma } ^ { 2 } + x ^ { 2 } ) ( s _ { \sigma } ^ { 2 } + 1 - v ^ { 2 } ) } \left[\exp \left(\alpha r_{2}(\varphi)\right)+\right.\right. \\
& \left.\left.+\exp \left(-i \alpha r_{2}(\varphi)\right)\right] d \varphi\right\}+ \\
& +\frac{1}{\sqrt{2} \pi} q_{0} x \operatorname{Im}\left\{e ^ { i \tau } \int _ { 0 } ^ { ( \infty ) } \frac { r _ { 2 } ( u ) \operatorname { e x p } ( - u \tau ) } { ( s _ { u } ^ { 2 } + \varkappa ^ { 2 } ) ( s _ { u } ^ { 2 } + 1 - v ^ { 2 } ) } \left[\exp \left(\alpha r_{2}(u)-i \frac{\pi}{4}\right)+\right.\right. \\
& \left.\left.+\sqrt{2} \exp \left(-i a r_{2}(u)\right)+\exp \left(-\alpha r_{2}(u)+i \frac{\pi}{4}\right)\right] d u\right\}- \\
& -\frac{q_{0} \sqrt{2}}{\left|\left(1-v^{2}-x^{2}\right)^{3 / 4}\right| \sqrt{\bar{\varepsilon}}} \exp \left[-a \sqrt{\left(1-v^{2}-\varkappa^{2}\right) / 2 \varepsilon}\right] \times \\
& \times \cos \alpha \sqrt{\left(1-v^{2}-x^{2}\right) / 2 \varepsilon} \cdot \sin x \tau \text {. }
\end{aligned}
$$

Области в плоскости $\left(\alpha,{ }^{\circ} \tau\right)$, где эта формула применима, различны для различных определенных интегралов. Относительно первого интеграла (выражающего действие контурной нагрузки $p(\tau)=p_{0} \sin x \tau$ ) возникает вопрос - определяет ли он достаточно точно прогиб; $w(\alpha, \tau)$ в некоторой узкой полосе за фронтом $(\alpha=\tau)$ продольной волны, обусловленный краевой нагрузкой $p(\tau)=p_{0} \sin x \tau$. Этот вопрос, однако, не имеет практического, значения, если главной задачей является определение наибольших амплитуд колебания. Область практической применимости третьего интеграла в (4.4) значительно у́же, так как $\operatorname{Re}_{2}(u)>0$ при $u>0$, и вычисление его связано при немалых значениях $\alpha / \tau$ с по- 
терей точности. Во избежание такого явления нужно деформировать контур интегрирования так, чтобы подинтегральная функция не была сильно осциллирующей. Эта процедура ведет к приложению метода перевала.

Представим приближенное выражение для прогиба от краевой нагрузки $q(\tau)=q_{0} \sin x \tau$ в форме

$$
\begin{aligned}
w(\alpha, \tau) & =\frac{q_{0}}{2 i \pi}\left\{\int_{C_{2}} G(s) F_{2 q}(s) H(s) e^{\alpha r_{2}(s)+s \tau} d s+\right. \\
+ & \left.\int_{C_{3}} G(s) F_{3 q}(s) H(s) e^{2 r_{3}(s)+s \tau} d s\right\} .
\end{aligned}
$$

Контур $C_{2}$ (фиг. 2) проводим через перевалы $s=s_{2}$ функцин $\operatorname{Re}\left[\alpha r_{2}(s)+\tau s\right]$, находящиеся на мнимой оси плоскости $s$. Отметим, что с увеличением отношения $\alpha / \tau$ увеличивается и $\left|s_{2}\right|$; при $\alpha / \tau=\kappa$ будет $s_{2} \approx \pm i \kappa / 3 \varepsilon$. По основному положению метода перевала на контуре $C_{2}$ должно быть $\operatorname{Im}\left[\alpha r_{2}(s)+\tau s\right]=\operatorname{Im}\left[\alpha r_{2}\left(s_{2}\right)+\tau s_{2}\right]$; соблюдать это правило можно до точки, где контур $C_{2}$ дойдет до вещественной оси, вдоль

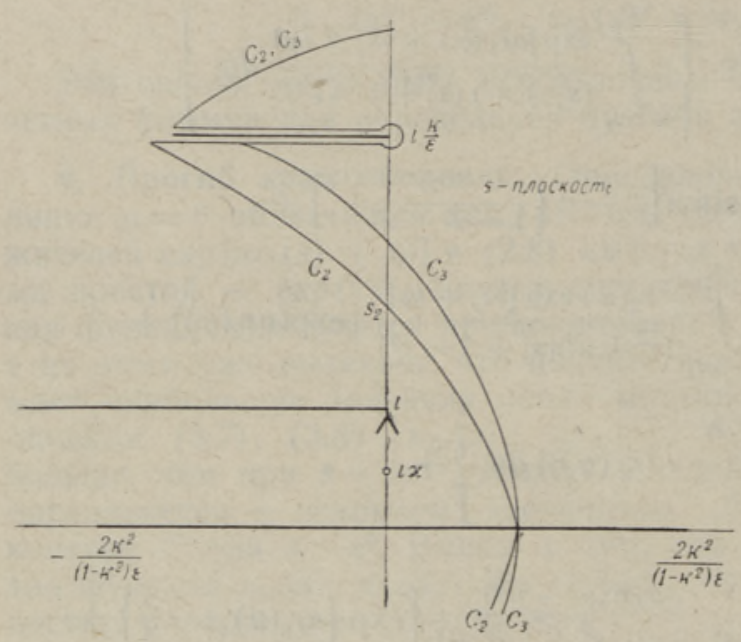

Фиг. 2. которой имеется срез (постоянные $\operatorname{Im}\left[\alpha r_{2}\left(s_{2}\right)+\tau s_{2}\right]$ на верхней и нижней полуплоскости по абсолютнсй величине равны, но противоположного знака). Из этой точки проводим контур $C_{3}$ по линии наискорейшего спуска функции $\operatorname{Re}\left[\alpha r_{3}(s)+\right.$ $+\tau s]$. Определенный таким образом контур $C_{3}$ с увеличением отношения $\alpha / \tau$ приблизится к контуру $C_{2}$. Но на берегу среза по вещественной оси $r_{3}(s)=\overline{r_{2}(s)}$, и сразу же вытекает альтернатива: либо нужно отказаться от применения метода перевала, так как точка контура $C$ на вещественной

оси слишком близка к точке перевала $s_{2}$ и функция ехр $\left[\alpha r_{2}(s)+\tau s\right]$ не успеет в достаточной мере затухнуть, либо второй интеграл в (4.5) по контуру $C_{3}$ мал по сравнению с первым и его можно не учитывать.

В узком секторе плоскости $(\alpha, \tau)$, где удовлетворяются сильные неравенства $\alpha^{2} / \tau^{2} \ll 1, \varepsilon^{2} \tau^{4} / \alpha^{4} \ll 1$, получим для (4.5) методом перевала следующую приближенную формулу:

$$
w(\alpha, \tau)=64 q_{0} \varkappa\left(\frac{2 \tau}{\pi \alpha^{2}}\right)^{1 / 2}\left(\sqrt{\varepsilon} \frac{\tau}{a}\right)^{5} \sin \frac{a^{2}}{4 \varepsilon \tau} .
$$

Из этой формулы следует, что в рассматриваемой области прогиб оболочки от действия краевой нагрузки $q(\tau)=q_{0} \sin \varkappa \tau$ является сильно осциллирующей функцией, амплитуда которой значительно меньше, чем $\max w(0, \tau) \mid$. Более того, значительно меньше в этой области и амплитуда $\partial^{2} w / \partial \alpha^{2}$ по сравнению с $\max \cdot \partial^{2} w(0, \tau) / \partial \alpha^{2} \mid$. Отсюда можно заключить, что исследование решения в области $(\alpha, \tau)$, где не выполняется сильное неравенство $\alpha^{2} / \tau^{2} \ll 1$ (а это будет широкий сектор за фронтом распространения упругого возмущения, $\alpha=\tau)$, имеет практиче- 
ское значение лишь в редких случаях. Надо прибавить, что исследование решения в указанной области отнюдь не просто: при вычислении определенных интегралов (2.8) нельзя пренебречь интегралами, взятыми по берегам среза $-\infty+i \kappa / \varepsilon, 0+i \kappa / \varepsilon$ (фиг. 1) и т. д., а подинтегральные функции также не допускают упрощения.

5. Упрощенные задачи для определения больших прогибов. Первые два члена формулы (4.4), выражающие действие краевой нагрузки $p(\tau)=p_{0} \sin \varkappa \tau$, могут быть получены как решение упрощенной системы уравнений преобразованием Лапласа

$$
\begin{aligned}
& \frac{\partial^{2} u}{\partial a^{2}}-\frac{\partial^{2} u}{\partial \tau^{2}}-v \frac{\partial w}{\partial a}=0 \\
& v \frac{\partial u}{\partial a}-\frac{\partial^{2} w}{\partial \tau^{2}}-w=0
\end{aligned}
$$

удовлетворяющее краевому условию

$$
\frac{\partial u}{\partial \alpha}-v w=p(\tau) \quad \text { при } \alpha=0
$$

и начальным условиям

$$
u(\alpha, 0)=w(\alpha, 0)=\frac{\partial}{\partial \tau} u(\alpha, 0)=\frac{\partial}{\partial \tau} w(\alpha, 0)=0
$$

кроме того, $u(\alpha, \tau)=w(\alpha, \tau) \equiv 0$ при $\alpha>\tau$.

Весьма вероятно, что решение этой системы не аппроксимирует достаточно хорошо решение исходной задачи (1.1) - (1.5) при $q_{0}=0$ в некоторой узкой полосе за фронтом волны $(\alpha, \tau)$. Однако, если задачей является определение наибольших прогибов оболочки, то этот недостаток не имеет существенного практического значения.

Последние три члена в формуле (4.4), выражающие прогиб от краевой нагрузки $q(\tau)=q_{0} \sin \varkappa \tau$, могут быть получены путем решения уравнения

$$
\varepsilon^{2} \frac{\partial^{4} w}{\partial c^{4}}+\frac{\partial 2 w}{\partial \tau^{2}}+\left(1-v^{2}\right) w=0
$$

удовлетворяющего при $\alpha=0$ краевым условиям

$$
-\varepsilon^{2} \frac{\partial 3 w}{\partial c^{3}}=q(\tau), \quad-\varepsilon \frac{\partial^{2} w}{\partial a^{2}}=m(\tau) \equiv 0
$$

и начальным условиям

$$
w(a, 0)=\frac{\partial}{\partial \tau} w(a, 0)=0
$$

Кроме того, при заданном $\tau$ функция $(\alpha, \tau)$ должна оставаться ограниченной, когда $\alpha \rightarrow \infty$.

Упрощенные соотношения (5.4) - (5.6) получаются из исходных соотношений $(1.1)-(1.4)$ следующим путем: в уравнениях (1.1), (1.3) пренебрегают членами $\partial^{2} u / \partial \tau^{2}, \partial^{2} v / \partial \tau^{2}$ соответственно, в (1.3) принимается $\partial^{2} v / \partial \alpha^{2}=-\varepsilon \partial^{3} w / \partial \alpha^{3}$ (по гипотезе Кирхгоффа-Лява $v=$ $=-\varepsilon \partial w / \partial \alpha)$, а в (1.2) полагается $\partial u / \partial \alpha=v w-$ это соотношение является результатом интегрирования упрощенного уравнения (1.1); вывод краевых условий (5.5) пояснений не требует. 
Решение упрощенной задачи (5.4) - (5.6) аппроксимирует решение исходной задачи $(1.1)-(1.5)$ при $p(\tau)=m(\tau) \equiv 0$ в некотором секторе у края $\alpha=0$, где $\alpha^{2} / \tau^{2} \ll 1$, а $\tau$ не слишком мало (при малых значениях $\tau$ решение не удовлетворяет условию $\partial^{2} w / \partial \tau^{2} \ll \partial^{2} w / \partial \alpha^{2}-$ основному положению упрощения).

6. Об обобщении результатов. Закончив рассмотрение данной конкретной задачи, хочется привести некоторые замечания о возможностях обобщения полученных результатов.

$1^{\circ}$. В разделе 1 было введено ограничение, по которому рассматривались только значения $x<\sqrt{1-v^{2}}$. Это предположение не является необходимым условием для применения соотношений (5.1) - (5.6): нужно требовать лишь, чтобы корни уравнения

$$
\varepsilon^{2} r^{4}-x^{2}+1-v^{2}=0
$$

достаточно хорошо аппроксимировали четыре корня уравнения (2.2) при $s=i \varkappa$, т. е. чтобы $х \varepsilon \ll 1$.

$2^{\circ}$. В работе рассматривались случаи, где на краю $\alpha=0$ приложены только осевая сила $p(\tau)=p_{0} \sin x \tau$ и поперечная сила $q(\tau)=q_{0} \sin x \tau$, а изгибающий момент $m(\tau)$ равен нулю. Если исследовать также случай $p(\tau)=q(\tau) \equiv 0, m(\tau)=m_{0} \sin x \tau$, то выясняется. что общие выводы и расчетные формулы будут такого же типа и содержания, как и в случае $p \equiv 0, m \equiv 0, q=q_{0} \sin x \tau$. Таким образом, при этих условиях динамика оболочки в краевой зоне (за исключением опять-таки некоторого начального промежутка времени) может быть определена при помощи упрощенного дифференциального уравнения (5.4), решение которого удовлетворяет при $\alpha=0$ краевым условиям

$$
-\varepsilon^{2} \frac{\partial 3 w}{\partial c^{3}}=0, \quad-\varepsilon \frac{\partial 2 w}{\partial c^{2}}=m_{0} \sin \varkappa \tau
$$

и начальным условиям (5.6).

$3^{\circ}$. Изменение краевых воздействий $p(\tau), q(\tau), m(\tau)$ во времени по закону $\sin x \tau$ не является необходимым условием для того, чтобы возможно было произвести расчет по изложенному упрощенному методу. Нужно лишь требовать, чтобы функции $p(\tau), q(\tau), m(\tau)$ имели небольшой показатель изменяемости (в вышеизложенной задаче это выражалось условием $\varepsilon x \ll 1)$ и чтобы $q(0), m(0)$ были малы по сравнению с наибольшими значениями $|q(\tau)|,|m(\tau)|$ в рассматриваемом промежутке времени.

Отметим, что в случае ступенчатого изменения нагрузки (например, $p(\tau) \equiv 0, q(\tau)=q_{0}=$ const, $m(\tau)=m_{0}=$ const при $\left.\tau>0\right)$ практический интерес представляет только исследование начальной стадии деформации. Правильно описать динамику оболочки в этой стадии упрощенное уравнение (5.4) не может.

4. Применение метода преобразования Лапласа для решения поставленной задачи показало, что для определения наибольших прогибов возможно использовать упрощенные соотношения - соотношения безмоментной теории и краевого эффекта статической теорни, дополненные инерционными членами. Если принять этот вывод за исходное положение и руководствоваться затем правилами полуобратного метода, то можно попытаться решить по упрощенным соотношениям осесимметрические динамические задачи и для конической оболочки вращения усеченного типа, выбирая подходящие численные методы решения этих упрощенных соотношений. 


\section{ЛИТЕРАТ У РА}

1. Э. И. Г ригол юк, О колебаниях пологой круговой цилиндрической панели, испытывающей конечные прогибы, Прикл. матем. и механ., 19, № 3, 1955.

2. Э. И. Гр иг ол юк, Нелинейные колебания и устойчивость пологих стержней и оболочек, Изв. АН СССР. Отд. техн. н., № 3, 1955.

3. А. С. В ольм и р, Об устойчивости цилиндрических оболочек при динамическом нагружении, Докл. АН СССР, 123, № 5, 1958.

4. В. Л. Агами ров, А. С. Вольми р, Поведение цилиндрических оболочек при динамическом приложении всестороннего давления или осевого сжатия, Изв. АН СССР. Отд. техн. н., Механика и машиностроение, № 3, 1959.

5. В. В. Болотин, Г. А. Бойченко, Б. П. Макаров, Н. П. Макаров, Н. И. С удаков а, Ю. Ю. Ш в ей к, О потере устойчивости тонких упругих оболочек под действием импульсивной нагрузки, Строит. механ. и расчет сооружений, 1, № 2, 1959.

6. Ю. Н. К ад ашевич, А. К. П е ц е в, О потере устойчивости цилиндрической оболочки при динамическом нагружении, Изв. АН, СССР. Отд. техн. н., Механика и машиностроение, № 3, 1960.

7. G. Hermann, I. M i r sky, Three-dimensional and shell-theory analysis of axially symmetric motions of cylinders. J. Appl. Mech., 23, No. 4, 1956.

Ннститут кибернетики

Академии наук Эстонской ССР

Поступила в редакцию

5. XI 1960

\section{PINGEOLUKORRA LAHUTAMISEST RINGSILINDRILISTE KOORIKUTE DUNAAMIKA TELGSOMMEETRILISTE OLESANNETE LAHENDAMISEL}

\section{N. Alumäe}

\section{Resümee}

Vaadeldakse pika suletud ringsilindrilise elastse kooriku deformatsiooni ühele servale rakendatud, ajas sinusoidaalselt muutuva koormise toimel. Piirdutakse dünaamika uurimisega paari esimese koormise muutumise perioodi vältel.

Artiklis näidatakse, et kui see periood on kas suurem või samas suurusjärgus kui aeg, mille vältel elastne laine läbib kauguse ja mis on võrdne kooriku keskpinna raadiusega, ja ülesanne seisneb paigutuste või pingete maksimaalsete väärtuste leidmises, siis on kooriku pinge- ja paigutusolek lahutatav momentidevabaks olekuks ja rajaefektiks. Need kvalitatiivselt täiesti erinevad pingeolekud on määratavad lihtsustatud vōrrandite abil, mis erinevad tasakaaluteooriast hästi tuntud võrranditest vaid inertsi arvestavate liikmete olemasolu tōttu.

Eesti NSV Teaduste Akadeemia

Küberneetika Instituut
Saabus toimetusse

5. XI 1960

\section{ON THE DECOMPOSITION OF THE STRESS-STATE IN AXISYMMETRICAL PROBLEMS OF MOTION FOR CIRCULAR CYLINDRICAL SHELL}

\section{N. Alumäe}

\section{Summary}

This paper considers the deformation of a long elastic circular cylinder under the action of edge forces sinusoidally varying in time.

The problem is presented by basic differential equations $(1.1)-(1.3)$ with boundary and initial conditions (1.4), (1.5) at loaded edge. Laplace transform method has been used to give the solution in the form of a contour integral. An analysis of this integral leads to the following conclusion: 1) if the period of the varying force is less or in order of the time necessary for the elastic wave to cover the distance equal to the radius of the middle surface of the shell, 2) the task is restricted to receive the maximum values of displacement components or stresses at first periods of loading, then the stress state of the shell can be separated to a membrane stress state and edge effect; these states can be determined by simplified equations which, apart from inertional terms, coincide with well-known equations of membrane stress and edge effect in problems of equilibrium. Academy of Sciences of the Estonian S.S.R.,
Institute of Cybernetics
Received

Nov. 5th, 1960 